\title{
Expected performance of the K-EUSO space-based observatory
}

\section{Francesco Fenu, ${ }^{a}, *$ Sergei Sharakin, ${ }^{b}$ Mikhail Zotov, ${ }^{b}$ Naoto Sakaki, ${ }^{c}$ Yoshiyuki Takizawa, ${ }^{c}$ Marta Bianciotto, ${ }^{a}$ Mario Bertaina, ${ }^{a}$ Marco Casolino ${ }^{c}$ and Pavel Klimov ${ }^{b}$ on behalf of the JEM-EUSO Collaboration}

(a complete list of authors can be found at the end of the proceedings)

${ }^{a}$ Università degli studi di Torino,

Via Pietro Giuria 1 10125, Torino, Italy

${ }^{b}$ Skobeltsyn Institute of Nuclear Physics,

Lomonosov Moscow State University, Russia

${ }^{c}$ RIKEN,

Wako, Saitama, Japan

${ }^{d}$ INFN, Structure of Rome Tor Vergata

Rome, Italy

E-mail: francesco.fenu@gmail.com

K-EUSO is a planned mission of the JEM-EUSO program for the study of ultra-high energy cosmic rays (UHECR) from space, to be deployed on the International Space Station. The K-EUSO observatory consists of a UV telescope with a wide field of view, which aims at the detection of fluorescence light emitted by extensive air showers (EAS) in the atmosphere. The EAS events will be sampled with a time resolution of $1-2.5 \mu$ s to reconstruct the entire shower profile with high precision. The detector consisting of $\sim 10^{5}$ independent pixels will allow a spatial resolution of $\sim 700 \mathrm{~m}$ on ground. From a $400 \mathrm{~km}$ altitude, K-EUSO will achieve a large and full sky exposure to sample the highest energy range of the UHECR spectrum. In this contribution, we present estimates of the performance of the observatory: an estimation of the expected exposure and triggered event rate as a function of energy and the event reconstruction performance, including resolution of arrival directions and energy of UHECRs.

$37^{\text {th }}$ International Cosmic Ray Conference (ICRC 2021)

July 12th - 23rd, 2021

Online - Berlin, Germany

\footnotetext{
${ }^{*}$ Presenter
} 


\section{Introduction}

The nature and origin of ultra-high energy cosmic rays (UHECRs) remains unsolved in contemporary astrophysics. The very low fluxes at extreme energies, of the order of few particles per $\mathrm{km}^{2}$ sr per century above $\sim 50 \mathrm{EeV}$, are a challenge for current observatories. The main goal of the Joint Experiment Missions for Extreme Universe Space Observatory (JEM-EUSO) program is the investigation of the UHECRs of the most extreme energies through the detection of fluorescence and Cherenkov light emitted by extensive air showers in the atmosphere [1]. From several hundred kilometers of altitude, such wide field of view telescopes will allow a very large exposure and therefore will probe the most extreme part of the spectrum.

K-EUSO is a fundamental cornerstone of the JEM-EUSO program. It is the first mission in this framework which will be capable of UHECR detection from space. It is planned to fly in 2024 and to be placed onboard the Russian segment of the International Space Station. The design presented here is a modified version of what was shown in [2] and is developed to fit the size and weight constraints imposed by the location on the International Space Station. The detector consists of a refractive optical system of $1400 \times 2400 \mathrm{~mm}^{2}$ size (see Fig. 1). The optics consists of two Fresnel lenses that focus the light onto a focal surface of $1300 \times 1000 \mathrm{~mm}^{2}$ size. The focal surface consists of 44 Photo Detector Modules (PDMs), of 36 Hamamatsu R11265-103-M64 Multi Anode Photomultipliers (MAPMTs) each. A MAPMT consists of 64 independent channels ( 8 per side) with a $3 \mathrm{~mm}$ size. Each of these channels (identified as pixels in the following) has a field of view of $0.1^{\circ}$ which corresponds to $\sim 700 \mathrm{~m}$ on the ground. The time resolution is in the process of definition and ranges from $1 \mu \mathrm{s}$ to $2.5 \mu \mathrm{s}$. This parameter will be determined as a trade-off between the limited hardware and telemetry budgets and the need of a good time resolution.



Figure 1: Scheme of the simulated K-EUSO detector.

The quantum efficiency of the MAPMTs is between 35 to $40 \%$ in the wavelength range 300 $400 \mathrm{~nm}$. The photomultiplier signal is read out and amplified by the SPACIROC3 ASIC [3] already in use in Mini-EUSO [4]. The SPACIROC3 operates in the single photoelectron mode and has a double pulse resolution of less than 10 ns. The majority of the data handling tasks, like data buffering, configuration of the read-out ASICS, triggering, synchronization and interfacing with the CPU system, is performed by the Zynq board. Given the very high time resolution of the detector $(\sim 1 \mathrm{MHz})$ and the high number of pixels, no full data retrieval is possible. Data must therefore satisfy strict trigger conditions. Concentrations of the signal are sought for by trigger algorithms 
to preferentially select the shower signal while rejecting background events. Data are saved on a hard drive, to be flown back to Earth, with a subset being sent by telemetry, as is being done now for Mini-EUSO.

In this work, we estimate the scientific performance of the K-EUSO detector. The study is done by means of simulations performed with the ESAF framework [5]. An example of a cosmic ray event simulated by ESAF is shown in Fig. 2. The top panel presents an example of a simulated photoelectron distribution of a cosmic ray shower (without any airglow emission). On the bottom left panel the same photoelectrons are plotted as a function of time. It can be seen that empty areas between MAPMTs on the focal surface cause a periodic decrease in the signal intensity. The bottom right panel shows the wavelength spectrum of the photons entering the detector. The fluorescence emission lines can be seen together with the Cherenkov continuum emission.
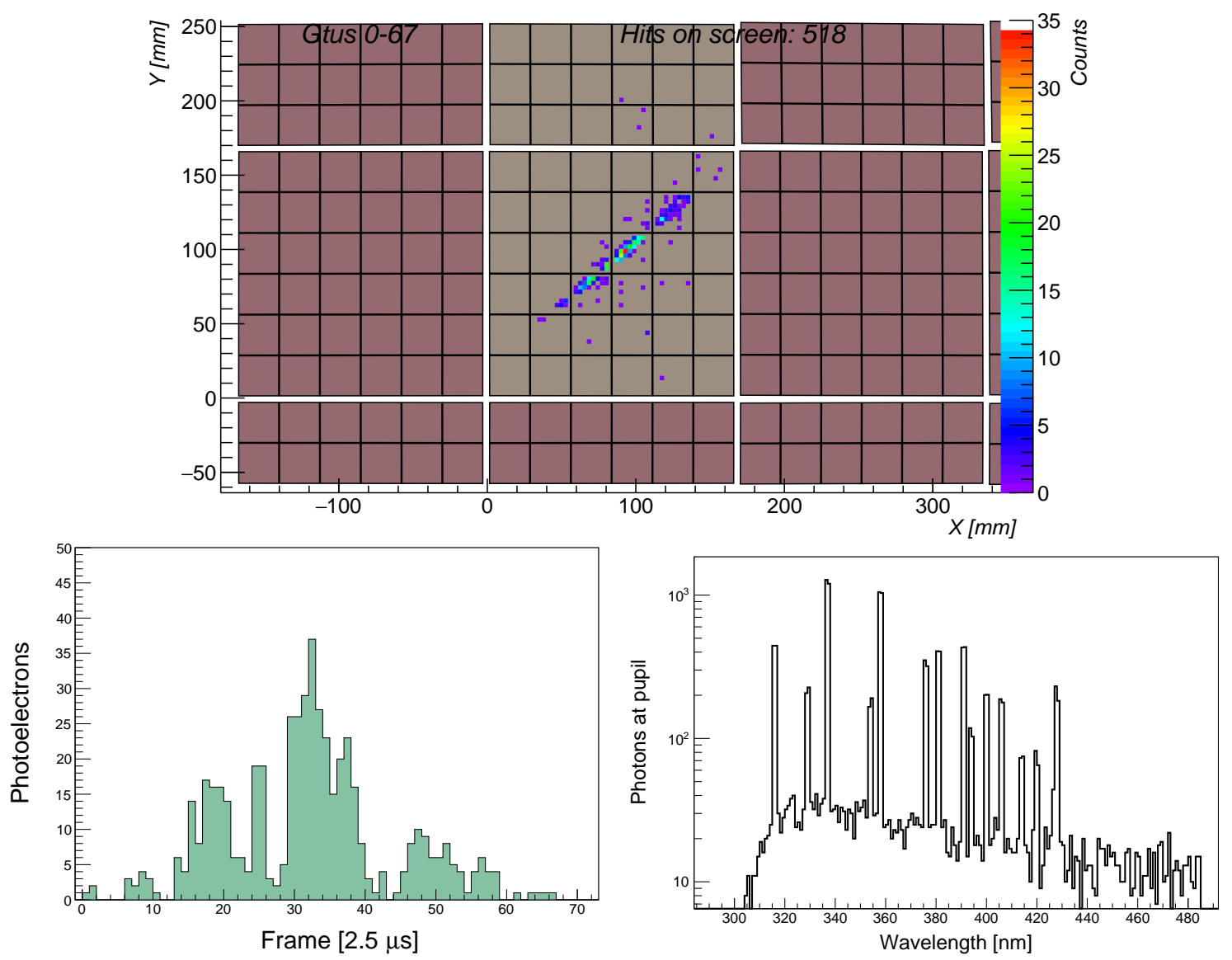

Figure 2: A $10^{20} \mathrm{eV}, 60^{\circ}$ event simulated by ESAF. Only signal from the shower is shown. On the top: the distribution of the signal generated by the shower on the focal surface. On the left: the corresponding simulated photoelectron time distribution. On the right: the spectrum of photons at the detector entrance.

\section{The K-EUSO exposure}

The trigger algorithms of the K-EUSO mission have been developed in the framework of the JEM-EUSO program [6] and are currently in the process of optimization. The logic is structured in 
multiple stages, each reducing the trigger rate by several orders of magnitude. The first level trigger, to be operated at the level of the PDM, looks for concentrations of the signal localized in space and time. The second level trigger is activated each time the first level trigger conditions are satisfied and integrates the signal intensity in a sequence of test directions. Directions consistent with one of the simulated extensive air showers have a higher chance of overcoming a preset threshold. Whenever such condition is met, the second level trigger is issued. The activation of the second level trigger starts the transmission and data storage procedure. The data acquisition is therefore stopped and data are either saved on a hard disk or sent to ground by telemetry. The trigger therefore reduces the data flow by several orders of magnitude. Thresholds are set to have a rate of the order of a trigger every few seconds at most to make the data acquisition consistent with the telemetry budget. The aim of this section is therefore to test the efficiency curve of the algorithm with respect to cosmic ray showers.

The exposure calculation is based on a Monte Carlo simulation of EAS of variable energy and direction. To avoid border effects, cosmic rays are injected in an area $A_{\text {simu }}$ larger than the field of view. The ratio of the triggered $N_{\text {trigg }}$ over simulated events $N_{\text {simu }}$ is then calculated for each energy bin. The solid angle $\Omega$ from which the cosmic rays arrive on the field of view is also included in the formula. The effects of day-night cycle and moon phases are taken into account in $\eta$, the astronomical duty cycle. Effects of clouds and artificial lights are also taken into account by $\eta_{\text {clouds }}$ and $\eta_{\text {city }}$ respectively. In this formula, we assumed $\eta=0.2, \eta_{\text {clouds }}=0.72$ and $\eta_{\text {city }}=0.9$ as estimated in [7]. The exposure $\mathscr{E}(E)$ is then calculated over time $t$, which is assumed to be 1 year in the following:

$$
\mathscr{E}(E)=\frac{N_{\text {trigg }}}{N_{\text {simu }}}(E) \times A_{\text {simu }} \times \Omega \times \eta \times \eta_{\text {clouds }} \times \eta_{\text {city }} \times t
$$

The yearly exposure as a function of energy is shown in Fig. 3. As it can be seen, at the plateau, which is reached at around $10^{20} \mathrm{eV}$, K-EUSO achieves an exposure of $\sim 18000 \mathrm{~km}^{2} \mathrm{sr} \mathrm{yr}$. The $50 \%$ efficiency is reached around $\sim 4 \times 10^{19} \mathrm{eV}$.

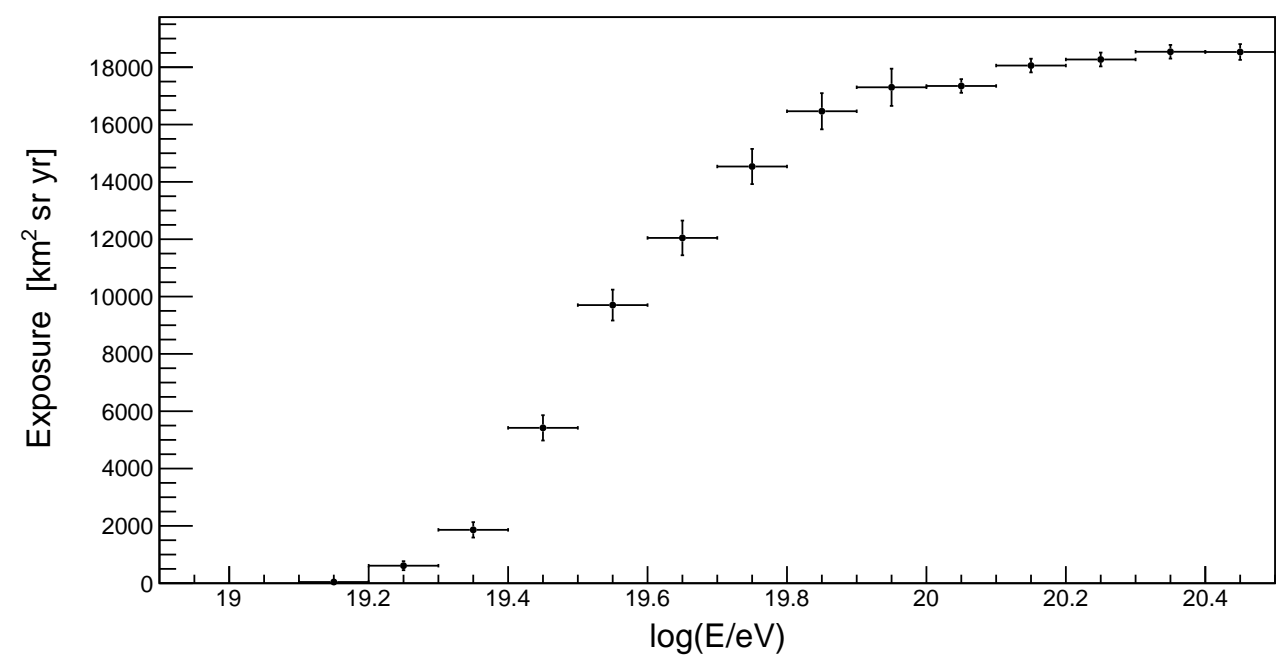

Figure 3: Annual exposure of K-EUSO as a function of UHECR primary energy. 
Assuming the spectrum recently published by the Auger collaboration [8], the expected rate of triggered events has been calculated to be of the order of 4 events/year above $10^{20} \mathrm{eV}$ and 65 events/year above $5 \times 10^{19} \mathrm{eV}$.

\section{The K-EUSO angular reconstruction}

At the occurrence of a trigger, the acquisition is stopped and data are retrieved. The information collected at this point is used then to reconstruct the parameters of the primary particle.

The first step consists in the recognition of the track, namely of pixels and frames in which the light of the shower arrives. A comprehensive review of the signal identification methods is given in [9] and [10]. All the methods look for concentrations of the signal in space and time that display kinematics consistent with the one of an extensive air shower.

The angular reconstruction extracts the arrival direction of the primary particle from the distribution and timing of the identified track. Several methods have been tested in the context of the JEM-EUSO program [9]. The method used for this work is based on a $\chi^{2}$ fit of the position and timing of the shower signal (the so called Numerical Exact 1 method). In this method the identification of the plane where both the shower and the detector lie is the first step of the procedure. The zenith angle of the shower is then reconstructed by comparing the arrival time of the photons from a test shower and the identified track. In Fig. 4, we show the reconstruction performance for arrival directions of EAS of $10^{20} \mathrm{eV}$ and zenith angles equal to $45^{\circ}$ and $60^{\circ}$ in the center of the field of view. To assess the quality of the reconstruction we plot the integral of the event distribution from 0 to a specific angle (in red).
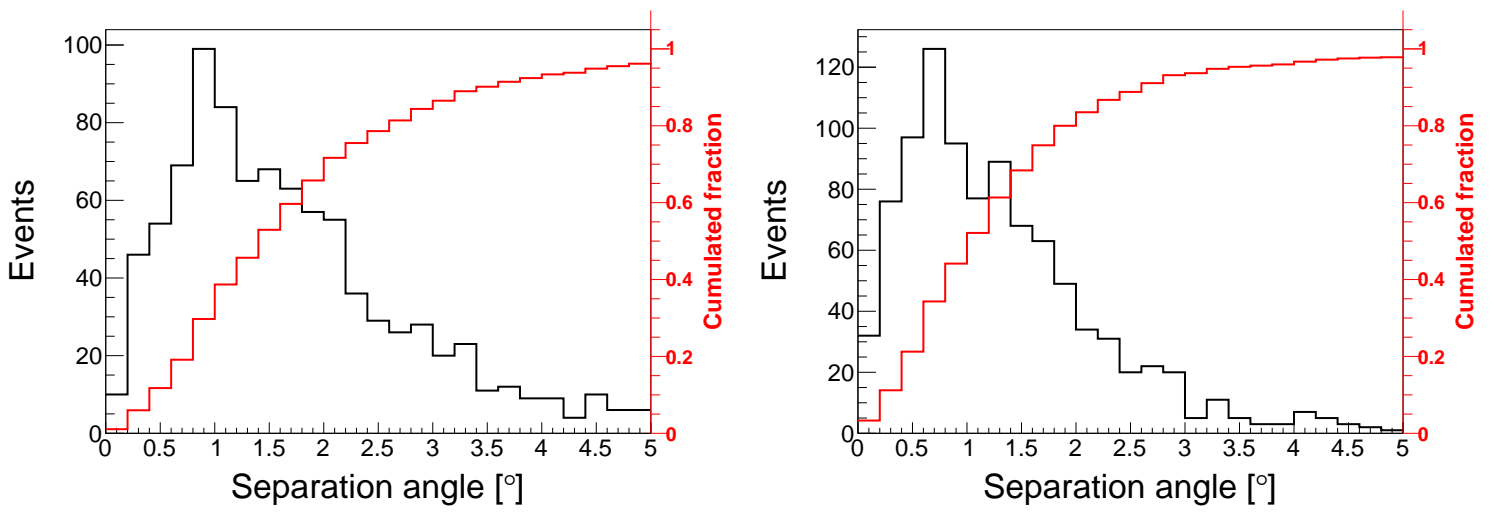

Figure 4: Performance of the angular reconstruction for UHECRs with the energy $10^{20} \mathrm{eV}$ in the center of the field of view. Left: $45^{\circ}$ zenith angle. Right: $60^{\circ}$ zenith angle.

In Fig. 5 the angular resolution is plotted as the angle within which $68 \%$ of the events fall. For this plot, we simulated 500 EASs in 16 different combinations of energy and zenith angle. For each condition, the events have been simulated over the entire field of view of the detector. It can be seen that K-EUSO achieves a resolution between 3 to 7 degrees at small zenith angles and improves to 1-2 degrees for nearly-horizontal events. There is a clear improvement trend as the energy increases. 


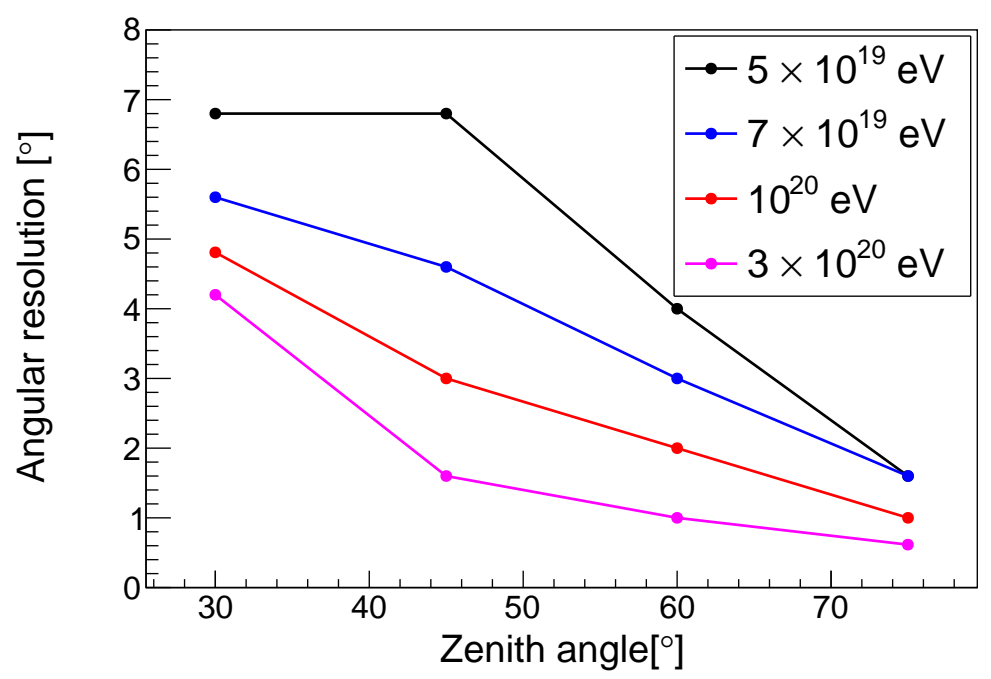

Figure 5: Estimated angular resolution of K-EUSO for different zenith angles and energies of a primary particle on the full field of view of the detector.

\section{The K-EUSO energy reconstruction}

The energy reconstruction is done according to [10] and is based on the signal identified by pattern recognition. The photoelectron profile is reconstructed based on the counts falling in the identified track with the airglow subtracted on average. The attenuation occurring in the detector is corrected following a look-up table relating an incident direction and wavelength of photons to the detector efficiency. Several methods to reconstruct the shower geometry have been implemented and are discussed in [9]. With an estimate of the position of the shower in atmosphere, it is possible to calculate the amount of atmospheric extinction and the luminosity curve. An estimate of the fluorescence yield is then used to reconstruct the charged particle profile of an EAS. Such profile is then fit with a shower profile parameterization to obtain the energy and the depth of maximum. An example of a $10^{20} \mathrm{eV}, 60$ degrees event reconstructed profile is shown in Fig. 6 as black crosses. A fit of the shower parameterization is shown in red. The reconstructed energy was $1.22 \times 10^{20} \mathrm{eV}$.

Estimations of the energy resolution of K-EUSO for UHECRs with different energies arriving at various zenith angles are shown in Fig. 7. As in the previous section, 2500 showers were simulated at fixed energies and zenith angles, both for the center and for the full field of view of the detector. The resolution was estimated as the standard deviation of the $\left(E_{\text {reco }}-E_{\text {real }}\right) / E_{\text {real }}$ distribution. It can be seen that the energy resolution is around $25 \%$ at low zenith angles and improves to around $15 \%$ for nearly horizontal events, with a small improvement trend toward higher energies ${ }^{1}$. No significant decrease of the performances has been observed if events are simulated on the full field of view.

\footnotetext{
${ }^{1}$ With the only exception of the $5 \times 10^{19} \mathrm{eV}, 30$ degrees condition. Here, at the threshold, the reconstruction is particularly challenging.
} 


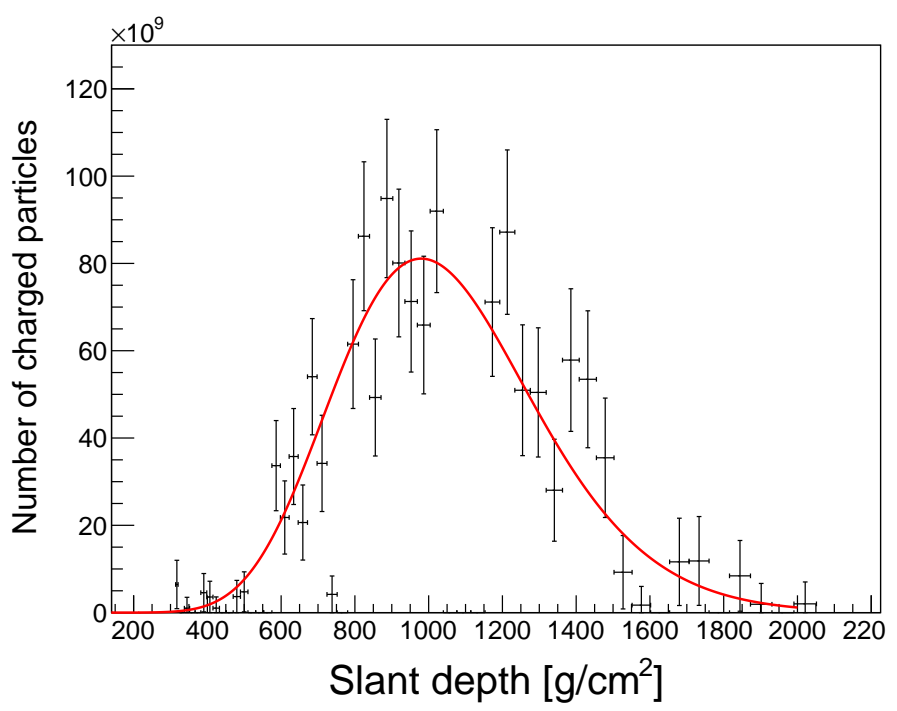

Figure 6: Reconstructed shower profile as a function of slant depth is shown as black crosses. The shower profile fit is shown in red. A shower generated by a $10^{20} \mathrm{eV}, 60$ degrees UHECR was simulated. The reconstructed energy equals $1.22 \times 10^{20} \mathrm{eV}$.
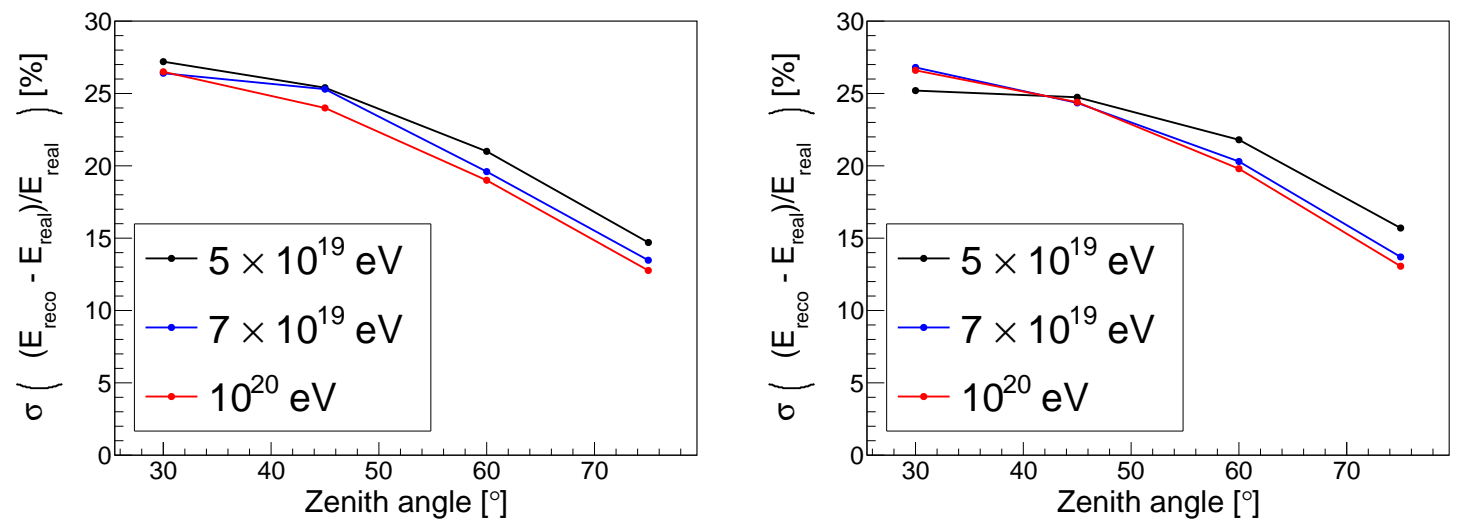

Figure 7: Estimates of energy reconstruction for different energy and zenith angle. Left: center of the field of view. Right: full field of view.

\section{Conclusions}

We presented estimations of the performance of the K-EUSO mission, which is a modified version of the "KLYPVE" project, aimed to meet weight and dimension constraints implied by its planned deployment at the Russian segment of the ISS. The expected rate of triggered events is around 65 per year for energies above $5 \times 10^{19} \mathrm{eV}$ and $\sim 4$ above $10^{20} \mathrm{eV}$ assuming the Pierre Auger Observatory spectrum. The angular reconstruction performance has also been studied for different conditions of energy and zenith angle on the whole field of view. The angular reconstruction varies from 3 to 7 degrees at low zenith angles and improves up to 1-2 degrees for nearly horizontal EAS. The higher energies are characterized by a better performance. The energy reconstruction has been tested for different conditions both in the center and on the whole field of view. The energy 
resolution was found to be around 25\% at low zenith angles and 15\% at higher angles. A mild trend of an improvement of the reconstruction performance is observed as the energy increases.

\section{Acknowledgments}

The authors express their deep and collegial thanks to the entire JEM-EUSO program and all its individual members. The article was prepared based on research materials carried out in the space experiment "KLYPVE," included in the Long-term program of Experiments on board the Russian Segment of the ISS. This work was supported by the State Space Corporation ROSCOSMOS by MAECI, the Italian Ministry of Foreign Affairs and International Cooperation, Projects of Major Relevance, by JSPS KAKENHI Grant (JP19H01915).

\section{References}

[1] M. Bertaina for the JEM-EUSO collaboration, "An overview of the JEM-EUSO program and results", Proc. of the $37^{\text {th }}$ International Cosmic Ray Conference, Berlin (2021)

[2] M. Casolino et al., "Observation of ultra high energy cosmic rays from space: status and perspectives.", Progr. of Theor. and Exp. Phys., Vol. 2017, 12, (2017)

[3] Blin et al., "SPACIROC3: a front-end readout ASIC for JEM-EUSO cosmic ray observatory.", Proc. of the $3^{r d}$ Int. Conf. on Technology and Instrumentation, Amsterdam (2014)

[4] S. Bacholle et al, "Mini-EUSO Mission to Study Earth UV Emissions on board the ISS", The Astroph. Jour. Supp. Series, Vol. 253, 2 (2021)

[5] Berat et al., "ESAF: Full Simulation of Space-Based Extensive Air Showers Detectors", Astroparticle Phys. Vol. 33, 4, P. 221-247 (2010)

[6] The JEM-EUSO Collaboration, "Cosmic ray oriented performance studies for the JEM-EUSO first level trigger", NIMA, 866, 150-163 (2017)

[7] The JEM-EUSO collaboration, "An evaluation of the exposure in nadir observation of the JEM-EUSO mission", Astroparticle Phys. 44, 76-90 (2013)

[8] The Pierre Auger Collaboration, "Measurement of the cosmic ray energy spectrum above $2.5 \times 10^{18} \mathrm{eV}$ using the Pierre Auger Observatory", Phys. Rev. D 102, 062005 (2020)

[9] The JEM-EUSO collaboration, "Performances of JEM-EUSO: angular reconstruction", Exp. Astr. 40, 153-177, (2015)

[10] The JEM-EUSO collaboration, "Performances of JEM-EUSO: energy and $X_{\max }$ reconstruction", Exp. Astr. 40, 183-214, (2015) 


\section{Full Authors List: JEM-EUSO Collaboration}

G. Abdellaoui ${ }^{a h}$, S. Abe ${ }^{f q}$, J.H. Adams Jr. ${ }^{p d}$, D. Allard ${ }^{c b}$, G. Alonso $^{m d}$, L. Anchordoqui ${ }^{p e}$, A. Anzalone $e^{e h, e d}$, E. Arnone $e^{e k, e l}$, K. Asano ${ }^{f e}$, R. Attallah ${ }^{a c}$, H. Attouiaa, M. Ave Pernas ${ }^{m c}$, M. Bagheri ${ }^{p h}$, J. Baláz ${ }^{l a}$, M. Bakiria ${ }^{a a}$ D. Barghini ${ }^{e l, e k}$, S. Bartocci ${ }^{e i, e j}$, M. Battisti ${ }^{e k, e l}$, J. Bayer ${ }^{d d}$, B. Beldjilali ${ }^{a h}$, T. Belenguer ${ }^{m b}$, N. Belkhalfa ${ }^{a a}$, R. Bellotti ${ }^{e a, e b}$, A.A. Belov ${ }^{k b}$, K. Benmessai ${ }^{a a}$, M. Bertaina ${ }^{e k, e l}$, P.F. Bertone ${ }^{p f}$, P.L. Biermann ${ }^{d b}$, F. Bisconti ${ }^{e l, e k}$, C. Blaksley ${ }^{f t}$, N. Blanc ${ }^{o a}$, S. BlinBondil $^{c a, c b}$, P. Bobik ${ }^{l a}$, M. Bogomilov $b a$, K. Bolmgren ${ }^{n a}$, E. Bozzo ${ }^{o b}$, S. Briz ${ }^{p b}$, A. Bruno ${ }^{e h, e d}$, K.S. Caballero ${ }^{h d}$, F. Cafagna ${ }^{e a}$, G. Cambiéei,ej, D. Campana ${ }^{e f}$, J-N. Capdevielle ${ }^{c b}$, F. Capel ${ }^{d e}$,

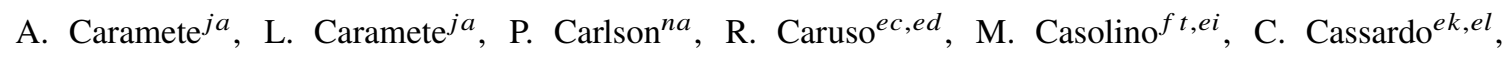

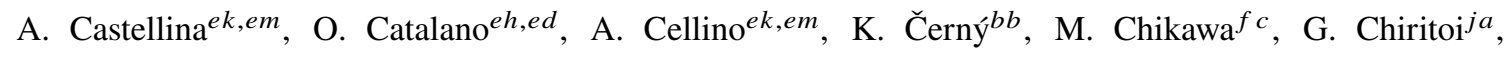

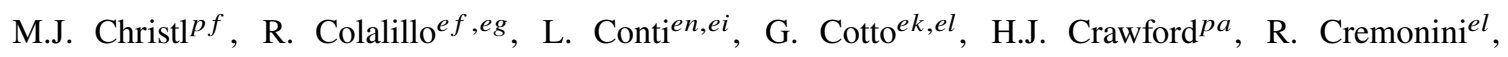
A. Creusot ${ }^{c b}$, A. de Castro Gónzalez ${ }^{p b}$, C. de la Taille ${ }^{c a}$, L. del Peral ${ }^{m c}$, A. Diaz Damian ${ }^{c c}$, R. Diesing ${ }^{p b}$, P. Dinaucourt ${ }^{c a}$, A. Djakonow ${ }^{i a}$, T. Djemil ${ }^{a c}$, A. Ebersoldt ${ }^{d b}$, T. Ebisuzaki ${ }^{f t}$, J. Eser ${ }^{p b}$, F. Fenu ${ }^{e k, e l}$, S. Fernández-González ${ }^{m a}$, S. Ferrarese ${ }^{e k, e l}$, G. Filippatos ${ }^{p c}$, W.I. Finch ${ }^{p c}$ C. Fornaro $^{e n, e i}$, M. Fouka $^{a b}$,

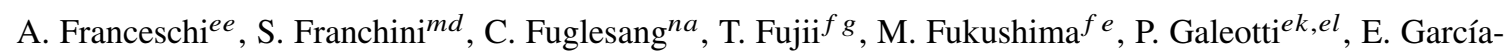
Ortega $^{m a}$, D. Gardiolek,em ${ }^{e k}$ G.K. Garipov ${ }^{k b}$, E. Gascón ${ }^{m a}$, E. Gazda ${ }^{p h}$, J. Gencil ${ }^{l b}$, A. Golzio ${ }^{e k, e l}$, C. González Alvarado ${ }^{m b}$, P. Gorodetzky ${ }^{f t}$, A. Green ${ }^{p c}$, F. Guarino ${ }^{e f, e g}$, C. Guépin ${ }^{p l}$, A. Guzmán ${ }^{d d}$,

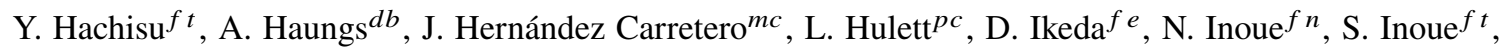

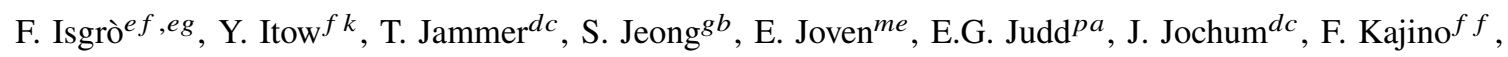
T. Kajino ${ }^{f i}$, S. Kalliaf, I. Kaneko ${ }^{f t}$, Y. Karadzhov $b a$, M. Kasztelan ${ }^{i a}$, K. Katahira ${ }^{f t}$, K. Kawai ${ }^{f t}$, Y. Kawasaki $^{f t}$, A. Kedadra ${ }^{a a}$, H. Khales ${ }^{a a}$, B.A. Khrenov ${ }^{k b}$, Jeong-Sook Kim ${ }^{g a}$, Soon-Wook Kim ${ }^{g a}$, M. Kleifges ${ }^{d b}$, P.A. Klimov ${ }^{k b}$, D. Kolev ${ }^{b a}$, I. Kreykenbohm ${ }^{d a}$, J.F. Krizmanic ${ }^{p f, p k}$, K. Królik ${ }^{i a}$, V. Kungel ${ }^{p c}$, Y. Kurihara ${ }^{f s}$, A. Kusenko ${ }^{f r, p e}$, E. Kuznetsov ${ }^{p d}$, H. Lahmar ${ }^{a a}$, F. Lakhdari ${ }^{a g}$, J. Licandro ${ }^{m e}$, L. López Campano ${ }^{m a}$, F. López Martínez ${ }^{p b}$, S. Mackovjak ${ }^{l a}$, M. Mahdi ${ }^{a a}$, D. Mandát ${ }^{b c}$, M. Manfrin ${ }^{e k, e l}$, L. Marcelli ${ }^{e i}$, J.L. Marcos ${ }^{m a}$, W. Marszał ${ }^{i a}$, Y. Martín ${ }^{m e}$, O. Martinez ${ }^{h c}$, K. Mase ${ }^{f a}$, R. Matev ${ }^{b a}$, J.N. Matthews ${ }^{p g}$, N. Mebarki ${ }^{a d}$, G. Medina-Tanco ${ }^{h a}$, A. Menshikov ${ }^{d b}$, A. Merino ${ }^{m a}$, M. Mese $^{e f, e g}$, J. Meseguer ${ }^{m d}$, S.S. Meyer ${ }^{p b}$, J. Mimouni ${ }^{a d}$, H. Miyamoto ${ }^{e k, e l}$, Y. Mizumoto ${ }^{f i}$, A. Monaco ${ }^{e a, e b}$,

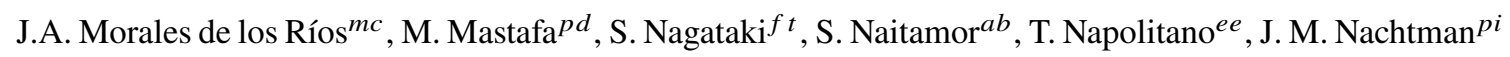


Y. Onel ${ }^{p i}$ G. Osteria ${ }^{e f}$, A.N. Otte ${ }^{p h}$, A. Pagliaro ${ }^{e h, e d}$, W. Painter ${ }^{d b}$, M.I. Panasyuk ${ }^{k b}$, B. Panico ${ }^{e f}$, E. Parizot ${ }^{c b}$, I.H. Park ${ }^{g b}$, B. Pastircak ${ }^{l a}$, T. Paul ${ }^{p e}$, M. Pech ${ }^{b b}$, I. Pérez-Grande ${ }^{m d}$, F. Perfetto ${ }^{e f}$, T. Peter $^{o c}$, P. Picozza ${ }^{e i, e j, f t}$, S. Pindado ${ }^{m d}$, L.W. Piotrowski ${ }^{i b}$, S. Piraino ${ }^{d d}$, Z. Plebaniak ${ }^{e k, e l, i a}$, A. Pollini ${ }^{o a}$, E.M. Popescu ${ }^{j a}$, R. Prevete ${ }^{e f}, e g$, G. Prévôt ${ }^{c b}$, H. Prieto ${ }^{m c}$, M. Przybylak ${ }^{i a}$, G. Puehlhofer ${ }^{d d}$, M. Putis ${ }^{l a}$, P. Reardon ${ }^{p d}$, M.H.. Reno ${ }^{p i}$, M. Reyes ${ }^{m e}$, M. Ricciee, M.D. Rodríguez Frías ${ }^{m c}$, O.F. Romero Matamala ${ }^{p h}$, F. Ronga ${ }^{e e}$, M.D. Sabau ${ }^{m b}$, G. Saccáec,ed , G. Sáez Cano ${ }^{m c}$, H. Sagawa ${ }^{f e}$, Z. Sahnoune ${ }^{a b}$, A. Saito ${ }^{f g}$, N. Sakaki ${ }^{f t}$, H. Salazar ${ }^{h c}$, J.C. Sanchez Balanzar ${ }^{h a}$, J.L. Sánchez ${ }^{m a}$, A. Santangelo $d d$, A. SanzAndrés $^{m d}$, M. Sanz Palomino ${ }^{m b}$, O.A. Saprykin ${ }^{k c}$, F. Sarazin ${ }^{p c}$, M. Sato ${ }^{f o}$, A. Scagliola ${ }^{e a, e b}$, T. Schanz ${ }^{d d}$, H. Schieler ${ }^{d b}$, P. Schovánek ${ }^{b c}$, V. Scotti ${ }^{e f, e g}$, M. Serra ${ }^{m e}$, S.A. Sharakin ${ }^{k b}$, H.M. Shimizu ${ }^{f j}$, K. Shinozaki ${ }^{i a}$, J.F. Soriano ${ }^{p e}$, A. Sotgiu ${ }^{e i, e j}$, I. Stan ${ }^{j a}$, I. Strharskýla, N. Sugiyama ${ }^{f j}$, D. Supanitsky ${ }^{h a}$,

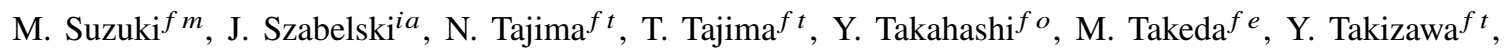
M.C. Talai ${ }^{a c}$, Y. Tameda ${ }^{f p}$, C. Tenzer ${ }^{d d}$, S.B. Thomas ${ }^{p g}$, O. Tibolla ${ }^{h e}$, L.G. Tkachev ${ }^{k a}$, T. Tomida ${ }^{f h}$,

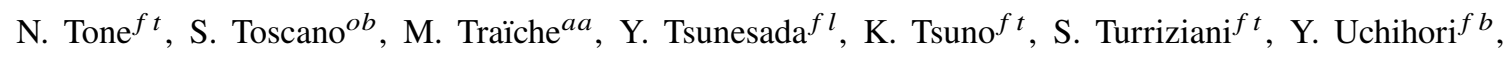


O. Vaduvescu ${ }^{m e}$, J.F. Valdés-Galicia ${ }^{h a}$, P. Vallania ${ }^{e k, e m}$, L. Valore ${ }^{e f, e g}$, G. Vankova-Kirilova ${ }^{b a}$,


J. Watanabe ${ }^{f i}$, J. Watts Jr. ${ }^{p d}$, R. Weigand Muñoz ${ }^{m a}$, A. Weindl ${ }^{d b}$, L. Wiencke ${ }^{p c}$, M. Wille ${ }^{d a}$, J. Wilms $^{d a}$, D. Winn ${ }^{p m}$ T. Yamamoto $f f$, J. Yang ${ }^{g b}$, H. Yano ${ }^{f m}$, I.V. Yashin ${ }^{k b}$, D. Yonetoku ${ }^{f d}$, S. Yoshida ${ }^{f a}$, R. Young ${ }^{p f}$, I.S Zgura ${ }^{j a}$, M.Yu. Zotov ${ }^{k b}$, A. Zuccaro Marchi ${ }^{f t}$

aa Centre for Development of Advanced Technologies (CDTA), Algiers, Algeria

${ }^{a b}$ Dep. Astronomy, Centre Res. Astronomy, Astrophysics and Geophysics (CRAAG), Algiers, Algeria

${ }^{a c}$ LPR at Dept. of Physics, Faculty of Sciences, University Badji Mokhtar, Annaba, Algeria

ad Lab. of Math. and Sub-Atomic Phys. (LPMPS), Univ. Constantine I, Constantine, Algeria

af Department of Physics, Faculty of Sciences, University of M'sila, M'sila, Algeria

ag Research Unit on Optics and Photonics, UROP-CDTA, Sétif, Algeria

${ }^{a h}$ Telecom Lab., Faculty of Technology, University Abou Bekr Belkaid, Tlemcen, Algeria

$b a$ St. Kliment Ohridski University of Sofia, Bulgaria

$b b$ Joint Laboratory of Optics, Faculty of Science, Palacký University, Olomouc, Czech Republic

$b c$ Institute of Physics of the Czech Academy of Sciences, Prague, Czech Republic

ca Omega, Ecole Polytechnique, CNRS/IN2P3, Palaiseau, France

${ }^{c b}$ Université de Paris, CNRS, AstroParticule et Cosmologie, F-75013 Paris, France

${ }^{c c}$ IRAP, Université de Toulouse, CNRS, Toulouse, France

da ECAP, University of Erlangen-Nuremberg, Germany

${ }^{d b}$ Karlsruhe Institute of Technology (KIT), Germany

$d c$ Experimental Physics Institute, Kepler Center, University of Tübingen, Germany

$d d$ Institute for Astronomy and Astrophysics, Kepler Center, University of Tübingen, Germany

de Technical University of Munich, Munich, Germany

ea Istituto Nazionale di Fisica Nucleare - Sezione di Bari, Italy

${ }^{e b}$ Universita' degli Studi di Bari Aldo Moro and INFN - Sezione di Bari, Italy

ec Dipartimento di Fisica e Astronomia "Ettore Majorana", Universita' di Catania, Italy

$e d$ Istituto Nazionale di Fisica Nucleare - Sezione di Catania, Italy

$e e$ Istituto Nazionale di Fisica Nucleare - Laboratori Nazionali di Frascati, Italy

ef Istituto Nazionale di Fisica Nucleare - Sezione di Napoli, Italy

eg Universita' di Napoli Federico II - Dipartimento di Fisica "Ettore Pancini", Italy

eh INAF - Istituto di Astrofisica Spaziale e Fisica Cosmica di Palermo, Italy

${ }^{e i}$ Istituto Nazionale di Fisica Nucleare - Sezione di Roma Tor Vergata, Italy

ej Universita' di Roma Tor Vergata - Dipartimento di Fisica, Roma, Italy

${ }^{e k}$ Istituto Nazionale di Fisica Nucleare - Sezione di Torino, Italy

el Dipartimento di Fisica, Universita' di Torino, Italy

em Osservatorio Astrofisico di Torino, Istituto Nazionale di Astrofisica, Italy

en Uninettuno University, Rome, Italy

$f$ a Chiba University, Chiba, Japan

$f b$ National Institutes for Quantum and Radiological Science and Technology (QST), Chiba, Japan

$f c$ Kindai University, Higashi-Osaka, Japan

$f d$ Kanazawa University, Kanazawa, Japan

$f e$ Institute for Cosmic Ray Research, University of Tokyo, Kashiwa, Japan 
$f f$ Konan University, Kobe, Japan

$f g$ Kyoto University, Kyoto, Japan

${ }^{f h}$ Shinshu University, Nagano, Japan

${ }^{f i}$ National Astronomical Observatory, Mitaka, Japan

$f j$ Nagoya University, Nagoya, Japan

$f k$ Institute for Space-Earth Environmental Research, Nagoya University, Nagoya, Japan

${ }^{f l}$ Graduate School of Science, Osaka City University, Japan

${ }^{f m}$ Institute of Space and Astronautical Science/JAXA, Sagamihara, Japan

${ }^{f n}$ Saitama University, Saitama, Japan

$f o$ Hokkaido University, Sapporo, Japan

$f p$ Osaka Electro-Communication University, Neyagawa, Japan

$f q$ Nihon University Chiyoda, Tokyo, Japan

$f r$ University of Tokyo, Tokyo, Japan

$f s$ High Energy Accelerator Research Organization (KEK), Tsukuba, Japan

${ }^{f t}$ RIKEN, Wako, Japan

${ }^{g} a$ Korea Astronomy and Space Science Institute (KASI), Daejeon, Republic of Korea

${ }^{g b}$ Sungkyunkwan University, Seoul, Republic of Korea

ha Universidad Nacional Autónoma de México (UNAM), Mexico

$h b$ Universidad Michoacana de San Nicolas de Hidalgo (UMSNH), Morelia, Mexico

$h c$ Benemérita Universidad Autónoma de Puebla (BUAP), Mexico

$h d$ Universidad Autónoma de Chiapas (UNACH), Chiapas, Mexico

he Centro Mesoamericano de Física Teórica (MCTP), Mexico

ia National Centre for Nuclear Research, Lodz, Poland

${ }^{i b}$ Faculty of Physics, University of Warsaw, Poland

${ }^{j a}$ Institute of Space Science ISS, Magurele, Romania

$k a$ Joint Institute for Nuclear Research, Dubna, Russia

$k b$ Skobeltsyn Institute of Nuclear Physics, Lomonosov Moscow State University, Russia

$k c$ Space Regatta Consortium, Korolev, Russia

la Institute of Experimental Physics, Kosice, Slovakia

$l b$ Technical University Kosice (TUKE), Kosice, Slovakia

ma Universidad de León (ULE), León, Spain

$m b$ Instituto Nacional de Técnica Aeroespacial (INTA), Madrid, Spain

${ }^{m c}$ Universidad de Alcalá (UAH), Madrid, Spain

$m d$ Universidad Politécnia de madrid (UPM), Madrid, Spain

me Instituto de Astrofísica de Canarias (IAC), Tenerife, Spain

na KTH Royal Institute of Technology, Stockholm, Sweden

oa Swiss Center for Electronics and Microtechnology (CSEM), Neuchâtel, Switzerland

$o b$ ISDC Data Centre for Astrophysics, Versoix, Switzerland

${ }^{o c}$ Institute for Atmospheric and Climate Science, ETH Zürich, Switzerland

pa Space Science Laboratory, University of California, Berkeley, CA, USA

${ }^{p b}$ University of Chicago, IL, USA

$p c$ Colorado School of Mines, Golden, CO, USA

$p d$ University of Alabama in Huntsville, Huntsville, AL; USA 
$p e$ Lehman College, City University of New York (CUNY), NY, USA

$p f$ NASA Marshall Space Flight Center, Huntsville, AL, USA

$p g$ University of Utah, Salt Lake City, UT, USA

$p h$ Georgia Institute of Technology, USA

${ }^{p i}$ University of Iowa, Iowa City, IA, USA

${ }^{p j}$ NASA Goddard Space Flight Center, Greenbelt, MD, USA

${ }^{p k}$ Center for Space Science \& Technology, University of Maryland, Baltimore County, Baltimore, MD, USA

${ }^{p l}$ Department of Astronomy, University of Maryland, College Park, MD, USA

${ }^{p m}$ Fairfield University, Fairfield, CT, USA 